\title{
Remember or not? For how long can a clonal plant remember drought stress?
}

\author{
Jiaxin Quan ${ }^{1}$, Zuzana Münzbergová ${ }^{2}$, and Vit Latzel ${ }^{3}$ \\ ${ }^{1}$ Northwest University \\ ${ }^{2}$ Botanicky ustav AV CR \\ ${ }^{3}$ Czech Academy of Sciences
}

June 24, 2021

\begin{abstract}
Stress can be remembered by plants in a form of 'stress memory' that can alter future phenotypes of previously stressed plants and even phenotypes of their offspring. It was shown that DNA methylation is among the mechanisms mediating the memory. It is not known for how long the memory is kept by plants. If the memory is long lasting, it can become maladaptive in situations when parental-offspring environment differ. We investigated for how long can a parental plant "remember" that it experienced a stress and pass the memory to its clonal offspring. We grew parental plants of three genotypes of Trifolium repens for five months either in control conditions or in control conditions that were interrupted with drought pulses applied for two months in four different time-slots. We also treated half of the parental plants with 5-azacytidine (5-azaC) to test for the potential role of DNA methylation in the stress memory. Then, we transplanted parental cuttings (ramets) individually to control environment and allowed them to produce offspring ramets for two months. The drought stress experienced by parents affected phenotypes of offspring ramets. Such a memory resulted in enhanced number of offspring side branches originating from plants that experienced drought stress maximally 6 weeks before their transplantation to control environment. We did not find any transgenerational memory in offspring of plants that experienced drought stress later than 6 weeks before their transplantation. 5-azaC also reduced the effect of transgenerational memory on offspring ramets. We confirmed that drought stress can trigger transgenerational memory in T. repens that is very likely mediated by DNA methylation. Most importantly, the memory was time limited and was gradually erased. We conclude that the time limited memory on environmental stress can be adaptive as climate tends to be variable and parental-offspring environmental conditions often do not match.
\end{abstract}

Remember or not? For how long can a clonal plant remember drought stress?

Jiaxin Quan ${ }^{1,2}$, Zuzana Münzbergová ${ }^{2,3}$, Vít Latzel $^{2 *}$

${ }^{1}$ Key Laboratory of Resource Biology and Biotechnology in Western China, Ministry of Education, Northwest University, Xi'an 710069, China

${ }^{2}$ Institute of Botany, Czech Academy of Sciences, Zámek 1, CZ-252 43 Pruhonice, Czech Republic

${ }^{3}$ Department of Botany, Faculty of Science, Charles University, Prague, Czech Republic

${ }^{*}$ Corresponding author: Vít Latzel; E-mail: vit.latzel@ibot.cas.cz

E-mail address for all authors: Jiaxin Quan, jiaxinquan007@163.com; Zuzana Münzbergová, zuzmun@natur.cuni.cz

\section{Abstract}

Stress can be remembered by plants in a form of 'stress memory' that can alter future phenotypes of previously stressed plants and even phenotypes of their offspring. It was shown that DNA methylation is 
among the mechanisms mediating the memory. It is not known for how long the memory is kept by plants. If the memory is long lasting, it can become maladaptive in situations when parental-offspring environment differ.

We investigated for how long can a parental plant "remember" that it experienced a stress and pass the memory to its clonal offspring. We grew parental plants of three genotypes of Trifolium repens for five months either in control conditions or in control conditions that were interrupted with drought pulses applied for two months in four different time-slots. We also treated half of the parental plants with 5-azacytidine (5-azaC) to test for the potential role of DNA methylation in the stress memory. Then, we transplanted parental cuttings (ramets) individually to control environment and allowed them to produce offspring ramets for two months.

The drought stress experienced by parents affected phenotypes of offspring ramets. Such a memory resulted in enhanced number of offspring side branches originating from plants that experienced drought stress maximally 6 weeks before their transplantation to control environment. We did not find any transgenerational memory in offspring of plants that experienced drought stress later than 6 weeks before their transplantation. 5 -azaC also reduced the effect of transgenerational memory on offspring ramets. We confirmed that drought stress can trigger transgenerational memory in T. repens that is very likely mediated by DNA methylation. Most importantly, the memory was time limited and was gradually erased. We conclude that the time limited memory on environmental stress can be adaptive as climate tends to be variable and parental-offspring environmental conditions often do not match.

Keywords Epigenetic memory; Memory persistence; DNA methylation; 5-azacytidine

\section{Introduction}

An increasing body of studies demonstrate that plants' exposure to different kinds of stresses in the past can affect their responses to the same and/or different stresses in the future and eventually prepare them to respond rapidly and/or adaptively to forthcoming stressful events (Bruce et al., 2007; Ding et al., 2013; Ramírez et al., 2015; Li et al., 2014, Iwasaki \& Paszkowski, 2014, Li et al., 2019). Such a phenomenon is commonly called 'stress memory' or "priming". In some cases, the stress memory can be passed to further generation(s) and affect thus offspring growth and response to the stress despite no direct experience with the stress (Cullins, 1973; Shock et al., 1998; Molinier et al., 2006; Monneveux et al., 2013; Trewavas, 2014). This transgenerational memory can allow for rapid adaptation to environmental condition if offspring environment resembles parental conditions (Mirouze \& Paszkowski, 2011; Latzel and Klimesova, 2010; Boyko \& Kovalchuk, 2011; Latzel et al., 2014; González et al., 2017; Crisp et al., 2016).

One of the intriguing questions is for how long can the str,ss memory persist in a plant? If the stress memory has physiological and/or phenotypic consequences and is maintained over long period, it could easily become maladaptive in situations when stress events are rare or even absent. Hence, the fundamental prerequisite for evolutionary adaptiveness of the stress memory must be its reversibility and transiency (Ding et al., 2012; Virlouvet et al., 2018). Memories on the experienced stress can be stored in the form of epigenetic variation (Bruce et al., 2007; Pascual et al., 2014; McIntyre \& Strauss, 2014; Richards et al., 2017). It has been shown that epigenetic memory can be transmitted to offspring generations (e.g. Verhoeven et al., 2010; Verhoeven \& van Gurp, 2012; González et al., 2018) and can be gradually lost after several sexual or asexual generations in the absence of the triggering environmental stress (Jiang et al., 2014; Shi et al., 2019). In the study of Shi et al. (2019) it took two years and ten clonal generations to reset most of the environmentally induced epigenetic memory in a clonal plant Alternanthera philoxeroides. The dynamic of environmental stress is, however, operating often at shorter time scales, usually within days or months. It is thus extremely interesting and important to focus on the stress memory from the temporal dynamic perspective in order to improve our understanding of stress memory in eco-evo processes in plants.

Drought is one of the main threats affecting plant growth, as water deficit affects plants at all levels from molecular, cellular, organ to the whole body (Li et al., 2014; Avramova, 2015; Li \& Liu, 2016; Tombesi et al., 2018). Studies have shown that plants that experienced repeated cycles of drought stress exhibited both transcriptional and physiological responses during a subsequent drought stress that were absent in 
plants without previous drought experience (Ding et al., 2012, 2014; Virlouvet et al., 2018). It has been also shown that the memory on drought can be passed to (a)sexual offspring in Oryza sativa, Trifolium repens, Arabidopsis thaliana or Zea mays(González et al., 2016; Li et al., 2019; Ding et al., 2012, 2014; Virlouvet et al., 2018) and can be even adaptive, i.e. offspring of stressed parents overcome the stress better than a naïve offspring (González et al., 2017). Clonal plants usually prefer wet habitats (Klimeš et al., 1997, van Groenendael et al., 1996; Ye et al., 2014) making them particularly vulnerable to drought events that should increase in their frequency and severity in the near future (Dai, 2012; Sherwood \& Fu, 2014).

Clonal plants may have greater ability to pass epigenetic information to asexual generations than non-clonal plants to sexual generation because of the lack of meiosis during clonal reproduction (Latzel \& Klimesova, 2010; Verhoeven \& Preite, 2014; Douhovnikoff \& Dodd, 2015; González et al., 2016; Paszkowski \& Grossniklaus, 2011; Latzel \& Münzbergová; 2018; Münzbergová et al., 2019). This makes clonal plants an ideal system for studying various ecological and evolutionary aspects of transgenerational stress memory in plants. Our previous studies on a clonal herb Trifolium repens have shown that it can develop genotype specific drought stress memory that is partly enabled by epigenetic mechanism, in this case by DNA methylation (González et al., 2016). We have also shown that the memory can be adaptive, i.e. offspring ramets of parents that experienced drought responded to the drought better than naïve offspring (González et al., 2017). The memory is translated into altered growth of offspring ramets in comparison to plants without the memory (González et al., 2016, 2017). In this study, we built on our previous studies on T. repens and tested for how long parental plant carries the stress memory that is detectable on clonal offspring phenotypes and whether the memory is co-facilitated by DNA methylation. We tested the following hypotheses: (1) Drought stress is altering growth of parental ramets. (2) This alternation triggers drought-stress memory that is time-limited and is reset after certain period since the last drought event. (3) The drought memory is facilitated by DNA methylation. Testing these hypotheses should enable us to put the stress memory phenomenon into a time frame context, which should improve our understanding of ecological and evolutionary consequences of stress memory in clonal plants.

\section{Materials and methods}

Plant material

We used Trifolium repens as the model in our study. It is a rapidly growing polycarpic perennial herb widely distributed in a variety of grasslands and pastures differing in soil type, nutrient level, and soil humidity (Burdon, 1983). Each phytomere of the plant consisting of a node, internode, subtending leaf, axillary bud, and two nodal root initials is usually considered as a ramet (Hay et al., 2001). Therefore, the growing stolon/branch is de facto consisting of interconnected ramets that can develop independent genet if connection between ramets is severed. We collected three cuttings taken from at least 50 meters distance from a mesophilous meadow of the park at the Institute of Botany, Pruhonice, Czech Republic to ensure that the three cuttings were of different genotypes but had similar growing conditions as well as growing history. We vegetatively propagated them for four months in the experimental garden prior the main experiment.

Study design

We conducted the experiment in a greenhouse at the Institute of Botany, Pruhonice, Czech Republic with controlled temperature and light regime from October 7, 2019 to May 4, 2020 (210 days in total). The greenhouse had controlled temperature $\left(23 / 18{ }^{\circ} \mathrm{C}\right.$ day/night $)$ and light regime $(12-/ 12$-h light/night cycle). The experiment was divided in two parts. The first consisted of stress memory induction in parental generation (further referred to as Parental generation), the second part was designed to test for how long the parental plant carries memory on the drought stress that affects clonal offspring generations (further referred to as Offspring generation).

\section{Parental generation:Drought stress application}

We created 120 standardized unbranched cuttings (parental ramets) from the pre-cultivated plant material (three genotypes, 40 cuttings per genotype) of T. repens . Each cutting consisted of three nodes with apical 
end and was planted individually into a tray $30 \times 40 \times 8 \mathrm{~cm}$ filled with standardized soil (Trávníkový substrát, AGRO CS a.s., Rikov, Czech Republic, mixture of sand, compost and peat, $75 \%$ mass water holding capacity). After transplantation of parental ramets, we kept all plants in control conditions for two weeks to allow recovery and successful rooting. The plants were grown for five months either in control conditions (watered when needed) or in control conditions repeatedly interrupted with drought pulses (watered only when leaves were wilting) that lasted for 10 weeks. We randomly assigned plants to five treatment combinations: control ( $\mathrm{n}=8$ per genotype), plants were watered regularly to keep the soil constantly moist during the whole cultivation period. Plants from the drought-stress treatments were divided in four groups in the way that each group experienced 10-week drought period in different time slots (2 weeks difference among the slots, see Fig. 1). In the first group ( $\mathrm{n}=8$ per genotype), the drought treatment ended 8 weeks before establishment of the Offspring generation part (further referred to as $\mathbf{8 W}$ group, see also Fig. 1). In the second group ( $\mathrm{n}=8$ per genotype), drought ended 6 weeks before establishment of the Offspring generation part (further referred to as $\mathbf{6} \mathbf{W}$ group). In the third group ( $\mathrm{n}=8$ per genotype), drought ended 4 weeks before establishment of the Offspring generation part (further referred to as $\mathbf{4 W}$ group). Finally, in the fourth group ( $\mathrm{n}=8$ per genotype), drought ended 2 weeks before establishment of the Offspring generation part (further referred to as $\mathbf{2} \mathbf{W}$ group). The drought stress was implemented by watering a plant with $200 \mathrm{ml}$ of water only when the plant showed significant drought stress response, i.e., most leaves wilting. The water volume that was determined by a pilot study sufficiently moistened the soil and ensured that the next drought pulse occurs within 4 to 7 days. During the 10-week drought period plants were watered approximately 10 times. The control plants received $8 \times$ (watered $2 \times$ more often with $4 \times$ more water volume at each watering occasion) more water than the drought stressed plants during the drought period. The same level of watering as in controls was maintained in the drought stressed plants outside the drought period. Two weeks after the last drought event (i.e. $140^{\text {th }}$ day of the experiment), we created single standardized cutting (4 nodes with apical end) from every plant individual. These cutting were used in the following Offspring generation part, see later. The remaining above ground biomass of parental plants was harvested, dried at $80^{\circ} \mathrm{C}$ for 48 hours and weighed. In a subset of randomly chosen plants we also checked the Rhizobia colonisation of roots. We did not find any established relationship in the 10 plants, which confirmed our previous experienced with the species that the Rhizobia colonisation is rare under our growing conditions.

\section{5-azacytidine application}

To test for the role of DNA methylation in the stress memory induced by drought, we applied 5-azacytidine demethylating agent on half of the parental plants, the remaining plants were sprayed with the same volume of pure water. 5-azacytidine (further referred to as 5-azaC) reduces the global cytosine methylation level of treated plants, and it has been successfully applied to demonstrate the role of plant epigenetic memory in plant adaptation to stress (e.g. Boyko et al., 2010; González et al., 2016). 5-azaC can be toxic to plants and thus some growth responses of plants can be consequences of the toxicity rather than the alteration of DNA methylation. The unwanted side effects of 5-azaC are, however, related almost exclusively to situations, when plants are germinated in 5-azaC solution (Puy et al. 2018). Foliar applications of 5 -azaC is bypassing most of the negative effects on plant growth but keeps its demethylating efficiency at comparable levels to germination plants in 5-azaC solution (Puy et al., 2018). We subjected a half of the parental plants to 5 -azaC treatment (4 plants per genotype and treatment) to alter their epigenetic memory. We regularly sprayed plants with $100 \mu \mathrm{mol}$ solution of 5 -azaC (Sigma-Aldrich, Praha, Czech Republic) every fourth day, which resulted in 32 spraying events. The first application was on October 21, 2019, i.e. 14 days after setting the experiment (the day of start of the first drought treatment), and with the last application at the time of the termination of the last drought treatment (February 10, 2020, $126^{\text {th }}$ day of the experiment). We sprayed the plants in early morning to ensure that plants had open stomata and the solution of 5-azaC could therefore be easily absorbed by the leaves.

\section{Offspring generation}

On day 140 of the experiment, we created single standardized cutting consisting of four nodes and apical end from each individual (40 cuttings per genotype, 120 cuttings in total) and transplanted them individually to 
similar trays filled with the same substrate as in the Parental generation. We cultivated them in a greenhouse under control condition for 10 weeks (from Day 140 to Day 210 of the experiment). We labelled the apical end of each transplanted cutting to be able to identify the end of parental (transplanted) ramet that had developed in the Parental generation and the new, offspring parts that have developed after transplantation (see Fig. 1b). During the 10 weeks' period, we recorded the length of the main stolon, number of nodes of the main stolon and number of side branches every week (10 times in total). This was used to calculate the growth rate of individual plants after transplantation. At the end of the experiment (Ten weeks after establishment of the Offspring generation), we harvested above-ground biomass separated in parental ramet planted into Offspring generation and offspring ramets (main stolon and side branches) that had developed after transplantation, dried them at $80^{\circ} \mathrm{C}$ for 48 hours and weighed.

\section{Statistical analyses}

We first tested the effect of genotype (genotype A, B and C), time since the last drought event $(2 \mathrm{~W}, 4 \mathrm{~W}$, $6 \mathrm{~W}, 8 \mathrm{~W}$ where $\mathrm{W}$ means week, and Control), 5-azaC application and their interactions on biomass of the plant grown in Parental generation of the experiment.

The data from continuous measurements of the plants in the Offspring generation were used to calculated growth rate based on stolon length, node number on main stolon and side branch number. To do this we fitted a growth function $\mathrm{a} \times \mathrm{b}^{\mathrm{x}}$ to the data using a non-linear least squares (nls) function in $\mathrm{R}$ 3.5.1. for each individual plant separately. The b value, representing the slope of the growth curve, was used as a measure of growth rate (Latzel et al. 2012).

Because we had too many performance measures and they were highly correlated to each other $(\mathrm{r}>0.7)$, we excluded some of the measures and retained only total biomass of the offspring (i.e. grown in Offspring generation), number of internodes of the main stolon, number of side branches and growth rate based on node number on main stolon and side branch number. We tested the effects of genotype, drought timing, 5-azaC application and their interactions on these plant performance measures. The parental biomass (biomass of the cutting transplanted to the Offspring generation that had developed before transplantation) was use as a covariate to account for potential initial size difference among transplanted ramets on the subsequent growth. All the tests were done using generalised linear model in R 3.5.1. Side branch number followed Poisson distribution. All the other variables followed Gaussian distribution. Total biomass and growth rate of side branch number had to be log transformed to fit the Gaussian distribution.

\section{Results}

\section{Parental generation}

Parental biomass differed among the genotypes (mean \pm SE, genotype A: $24.50 \mathrm{~g} \pm 16.45$; genotype B: 17.87 $\mathrm{g} \pm 13.55$; genotype C: $24.21 \mathrm{~g} \pm 17.06$ ) and was affected by the time period since the last drought (Table 1). Control plants were the biggest and the plants that received drought treatment in different time slots were almost identical (Fig S1, supplementary material). There was also a significant interaction between 5-azaC and time since the last drought (Table 1). Application of 5-azaC decreased the parental biomass in control but not in any of the drought stress treatments (Table 1, Fig. S1).

\section{Offspring generation}

The number of nodes on the main stolon, number of branches and growth rate of the main stolon and branches significantly differed among the genotypes (Table 1). Number of nodes on the main stolon, number of branches and growth rate of the main stolon and branches were also significantly affected by the time period since the last drought (Time since the last drought, Table 1). Biomass of offspring ramets produced by parental ramets that experienced last drought event 2, 4 and 6 weeks before transplantation was higher compared to offspring biomass of parental ramets from control conditions (Fig. 3, Table 2). Number of nodes of the main stolon was higher for offspring of parents that experienced last drought event 4 and 6 weeks before transplantation compared to offspring of other drought treatments (Table 2). Plants of parental ramets that experienced last drought event 4 weeks before transplantation produced more branches than plants of other 
parental drought groups (Table 1, Fig. 2). The growth rate of node number of the main stolon and number of branches was lower in plants created by parental ramets that experienced last drought event 2 and 8 weeks before transplantation in comparison to other treatments (Table 2).

\section{The effect of 5-azacytidine on Offspring generation}

Application of 5-azaC on parental plants reduced number of side branches in offspring plants (Table 1, Control:10.45 $\pm 0.37,5$-azaC: $8.33 \pm 0.37$ ), but did not have a main effect on the other measured variables in offspring. The effect of 5 -azaC interacted with time since last drought for node number and its growth rate and number of branches (Table 1, Fig. 2a, b). Drought stress in parental generation generally increased the number of nodes of the main stolon in Offspring generation, the 5-azaC significantly reduced number of the nodes in offspring of parents that experienced last drought event two weeks before transplantation (Fig. 2a). The application of 5-azaC in parents did not affect number of side branches produced by control parents (Fig. 2b), but reduced number of side branches in offspring of parents that experienced the last drought event 4 and 6 weeks before transplantation (Fig. 2b). Growth rate of the main stolon node number was interactively affected by the time since last drought and 5azaC. When 5-azaC was applied to parental ramets, the growth rate of offspring of parents that experienced the last drought event 2 and 8 weeks before transplantation significantly declined compared to offspring of control parents (Fig. 4; Fig S3).

\section{Discussion}

Our study investigated whether a clonal plantTrifolium repens can remember drought stress, and if so, for how long. We hypothesized that the memory should be gradually erased with the increasing time since the last drought experience. This prediction assumes that the long-term phenotypic consequences of the memory should be not beneficial in situation when the drought stress is infrequent or absent for a long period (Jiang et al., 2014; Shi et al. 2019; Lukic et al., 2020).

Results of our study are mostly in agreement with the predictions. We found that growth of offspring ramets was affected by the drought experienced by parents, which can be considered as an evidence of transgenerational stress memory. Such a memory was detected for offspring of parental ramets that experienced the last drought event 4 or 6 weeks before they were transplanted into the control environment in most of measured parameters but offspring biomass. Offspring biomass was affected even for offspring of parental ramets that experienced the last drought event 2 weeks before transplantation to control environment. Interestingly, some of the memory effects on the growth of offspring were absent in offspring of parents that were treated by 5 -azaC. Our results thus suggest that the drought in parental generation can trigger transgenerational memory that can be carried maximally six weeks by parents of T. repens. The drought memory was in majority of cases erased by application of 5-azaC indicating that DNA methylation was involved in the memory. Our results thus indirectly supported the mounting evidence that epigenetic processes are involved in stress memory in plants (e.g. Molinier et al., 2006; Boyko et al., 2007; Whittle et al., 2009; Verhoeven et al., 2010; Xu et al., 2016; Nakamura \& Hennig, 2017).

Some studies showed that the environmentally induced epigenetic change can be heritable among several sexual generations in the absence of the triggering stress (Verhoeven et al., 2010; Xu et al., 2016). Shi et al. (2019) showed that the environmentally induced epigenetic variation is degrading over 10 clonal generations (10 offspring ramets created from the establishment of the study) in a plant Alternanthera philoxeroides when cultivated in a common environment. These studies did not test the phenotypic consequences of epigenetic memory in plants but demonstrated that the environmentally induced epigenetic change can be heritable in certain cases (and species) and is carried by several (a)sexual generations. This is very intriguing phenomenon suggesting that some environments trigger memory that is fixed over longer period (and generations) than others and that the memory can be species specific. In our study, we simulated an environment that is repeatedly desiccating during summer season, i.e. periods with sufficient water supply were interrupted by periods of water shortage. This particular setting triggered memory that lasted for 6 weeks in the three genotypes of $T$. repens . Other scenarios with different timing and/or severity of a stress could trigger different memory effects that can have different phenotypic consequences on offspring generation. For instance, in our 
previous research on the same species, we observed that the stress memory is established only if the drought last for a certain period. We found that the drought stress can trigger transgenerational effects if it last for 10 weeks but not for 4 months (Rendina González et al., 2016). This phenomenon needs to be investigated in more detail to get better idea about the role of environmental stress, its intensity and duration on induction and temporal dynamics of stress memory in plants.

Previous studies investigated the role duration or intensity of environmental stress on induction of transgenerational effects (e.g. Boyko, 2010; Verhoeven \& van Gurp, 2012; Rahavi \& Kovalchuk 2013a, b; González et al. 2016; Racette et al., 2019) but did not consider the temporal dynamics of the stress memory. For instance, study by González et al. (2017) showed that the drought in parental generation can trigger adaptive stress memory in T. repens, i.e. offspring performed better in drought if their parents also experienced drought in comparison to offspring of naïve parents. However, they demonstrated it on offspring of parents that experienced drought period only recently, which may be ecologically rather rare scenario. It is possible that documented patterns of plant memory effects on transgenerational plasticity can be only snap shots in time, which can result in overestimation or underestimation of ecological and evolutionary aspects of memory in plants.

Our results along with other studies (Verhoeven \& van Gurp, 2012; González, 2016; Münzbergová et al. 2019, Racette et al., 2019) point also on the genotype specificity of the stress memory. The length and number of nodes of the main stolon (parental ramet) were significantly or marginally significantly affected by the drought timing, genotype and 5-azaC application (Table 1; Fig. S2). The inherent differences among genotypes support the assumption that the existence and mechanisms of parental stress memory vary between genotypes. Growth rate of parental stolon length was significantly affected by interaction between time since last drought and methylation (Table 1). Our results suggest a possibility that DNA methylation is associated with, and may partially regulate, growth of the main stolon under drought stress, through a dynamic alteration of methylation patterns.

\section{Other potential mechanisms involved in observed patterns of stress memory}

The more vigorous growth of offspring of parents that experienced the last drought event 2 to 6 weeks indicates that the stress memory cannot be ascribed to negative consequences of physiological damage of the parents but rather to other mechanisms including DNA methylation. The mostly comparable growth of offspring of parents that experienced the last drought event 2 weeks prior their transplantation to control environment with controls implies that the stress memory effect was likely to some degree downregulated by remaining negative physiological consequences of the not so long-ago experienced drought (e.g. Kannenberg et al., 2020). On the other hand, the comparable growth of offspring of parents that experienced last drought 8 weeks and controls can be best explained by the loss of the drought memory. Similar biomass produced by parents from drought treatments applied in different time slots indicates that the stress intensity was similar across the parental treatments despite parents experienced the stress in their slightly different developmental stages. This is a good indicator that the differing stress memory observed in offspring was not a consequence of different stress intensity in parents.

Conclusion

The term stress memory has been already well established in plant ecology and became commonly accepted by experts. Based on our results of the actual as well as previous studies (e.g. Rendina González et al., 2016), we argue that the next inevitable step in upcoming research should be involvement of the temporal dynamics of the stress memory from the perspective of stress duration and the time when the stress occurred. This can help us not only better understand ecological and evolutionary aspects of the memory in plants but could also improve our predictions of plant responses to future climatic conditions. More detailed insights into molecular (epigenetic) and biochemical mechanisms involved in the stress memory would also considerably improve our understanding of the memory mechanisms in our study. Although we focused on clonal generations, similar aspects of temporal dynamics of stress memory could be relevant for sexually derived individuals too.

\section{Author contributions}


V. Latzel and JX Quan conceived and designed the experiments. JX Quan performed the experiments, Z. Münzbergová helped the data analysis, JX Quan, V Latzel and Z. Münzbergová wrote the manuscript.

\section{Acknowledgements}

We are grateful to members of the Department of Population biology for comments on the previous version of the manuscript. This research was supported by the Czech Science Foundation project GAČR 17-11281S and partly by the institutional research project RVO 67985939 . JXQ was financially supported by the National Natural Science Foundation of China (31200249), National Special Program on Basic Works for Science and Technology of China (2015FY1103003-6).

\section{Data Accessibilty Statement}

Upon acceptance, we make sure to archive our data in a publicly accessible repository, such as Dryad.

\section{Competing Interests Statement}

No conflict of interest exits in the submission of this manuscript, and manuscript is approved by all authors for publication.

\section{References}

Avramova, Z. (2015). Transcriptional 'memory' of a stress; transient chromatin and memory (epigenetic) marks at stress response genes. Plant Journal , 83, 149-59.

Bossdorf, O., Richards, C. L., \& Pigliucci, M. (2008). Epigenetics for ecologists. Ecology Letters, 11 (2), 106-115.

Boyko, A., Kathiria, P., Zemp, F. J., Yao, Y. L., Pogribny, I., \& Kovalchuk, I. (2007). Transgenerational changes in the genome stability and methylation in pathogen-in-fected plants (Virus-induced plant genome instability). Nucleic Acids Research , 35, 1714-1725.

Boyko, A., Blevins, T., Yao, Y. L., Golubov, A., Bilichak, A., Ilnytskyy, Y., Hollander, J., Meins, F., \& Kovalchuk, I. (2010). Transgenerational adaptation of Arabidopsis to stress requires DNA methylation and the function of DICER-LIKE proteins. PLoS ONE , 5, e9514.

Boyko, A., \& Kovalchuk, I. (2011). Genome instability and epigenetic modification- heritable responses to environmental stress? Current Opinion Plant Biology , 14, 260-266.

Bruce, T. J., Matthes, M. C., Napier, J. A., \& Pickett, J. A. (2007). Stressful "memories" of plants: evidence and possible mechanisms.Plant Science, 173, 603-608.

Burdon, J. J. (1983). Biological flora of the British Isles: Trifolium repens.Journal of Ecology , 71 : 307-330.

Crisp, P. A., Ganguly, D., Eichten, S. R., Borevitz, J. O., \& Pogson, B. J. (2016). Reconsidering plant memory: intersections between stress recovery, RNA turnover, and epigenetics. Science Advances ,2 , 1-14.

Cullins, C. A. (1973). DNA differences between flax genotrophs. Nature ,243, 515-516.

Dai, A. (2012). Increasing drought under global warming in observations and models. Nature Climate Change , 3, 52 .

Ding, Y., Fromm, M., \& Avramova, Z. (2012). Multiple exposures to drought "train" transcriptional responses in Arabidopsis.Nature Communications , 3, 740.

Ding, Y., Liu, N., Virlouvet, L., Riethoven, J. J., Fromm, M., \& Avramova, Z. (2013). Four distinct types of dehydration stress memory genes inArabidopsis thaliana . BMC Plant Biology , 13, 229.

Ding, Y., Virlouvet, L., Liu, N., Riethoven, J. J., Fromm, M., \& Avramova, Z. (2014). Dehydration stress memory genes of Zea mays ; comparison with Arabidopsis thaliana . BMC Plant Biology, 14, 141. 
Douhovnikoff, V., \& Dodd, R. S. (2015). Epigenetics: a potential mechanism for clonal plant success. Plant Ecology , 216 (2), 227-233.

González, R. A. P., Chrtek, J., Dobrev, P. I., Dumalasová, V., Fehrer, J., Latzel, V., \& Mráz, P. (2016). Stress-induced memory alters growth of clonal offspring of white clover (Trifolium repens ).American Journal of Botany , 103 (9), 1567-1574.

González, R. A. P., Dumalasová, V., Rosenthal, J., Skuhrovec, J., \& Latzel, V. (2017). The role of transgenerational effects in adaptation of clonal offspring of white clover (Trifolium repens ) to drought and herbivory.Evolutionary Ecology , 31 (3), 345-361.

van Groenendael, J. M., Klimeš, L., Klimešová, J., \& Hendriks, R. J. J. (1996) Comparative ecology of clonal plants.PhilosophicalTransactions of theRoyalSociety B-Biological Sciences , 351 : 1331-1339.

Hay, M. J. M., Newton, P. C. D., Robin, C., \& Cresswell, A. (2001). Branching responses of a plagiotropic clonal herb to localised incidence of light simulating that reflected from vegetation. Oecologia , 127, 185-190.

Iwasaki, M., \& Paszkowski, J. (2014). Epigenetic memory in plants.EMBO Journal , 33 (18), 1987-1998.

Jiang, J., Qian, Q, Ma, B. J., \& Gao, Z. Y. (2014). Epigenetic variation and its application in crop improvement. Hereditas , 36 (5), 469-475. (in Chinese with English abstract)

Klimeš, L., Klimešová, J., \& Hendriks, R. (1997) Clonal plant architecture: A comparative analysis of form and function. In: de Kroon, H., van Groenendael, J.M., editors. The ecology and evolution of clonal plants. Leiden: Backhuys, pp. 1-29.

Latzel, V., \& Klimešová, J. (2010). Year-to-year changes in expression of maternal effects in perennial plants.Basic and Applied Ecology, 11 (8), 702-708.

Latzel, V., Zhang, Y., Moritz, K. K., Fischer, M., \& Bossdorf, O. (2012) Epigenetic variation in plant responses to defence hormones. Annals of Botany , 110 , 1423-1428.

Latzel, V., Janeček, Š., Doležal, J., Klimešová, J., \& Bossdorf, O. (2014). Adaptive transgenerational plasticity in the perennialPlantago lanceolate. Oikos , 123 (1), 41-46

Latzel, V., \& Münzbergová, Z. (2018). Anticipatory behavior of the clonal plant Fragaria vesca . Frontiers in Plant Science ,9 .

Li, X. N, \& Liu F. L. (2016). Drought stress memory and drought stress tolerance in plants: biochemical and molecular basis.DroughtStressTolerance in Plants , 1 , 17-44.

Li, P., Yang, H., Wang, L., Liu, H., Huo, H., Zhang, C., Liu, L. Liu A., Zhu A., Hu j., \& Lin Y. (2019). Physiological and transcriptome analyses reveal short-term responses and formation of memory under drought stress in rice.Frontiers in Genetices, 10 .

Li, X., Cai, J., Liu, F., Dai, T., Cao, W., \& Jiang, D. (2014). Cold priming drives the sub-cellular antioxidant systems to protect photosynthetic electron transport against subsequent low temperature stress in winter wheat. Plant Physiology Biochemistry ,82, 34-43.

Lukic, N., Kukavica, B., Davidovic-Plavsic, B., Hasanagic, D., \& Walter J. (2020). Plant stress memory is linked to high levels of anti-oxidative enzymes over several weeks, Environmental and Experimental Botany, $178,104166$.

Mcintyre, P. J., \& Strauss, S. Y. (2014). Phenotypic and transgenerational plasticity promote local adaptation to sun and shade environments.Ecology and Evolution , 28 (2), 229-246.

Mirouze, M., \& Paszkowski, J. (2011). Epigenetic contribution to stress adaptation in plants. Current Opinion Plant Biology , 14, 267-274. 
Molinier, J., Ries, G., Zipfel, C. \& Hohn, B. (2006). Transgeneration memory of stress in plants. Nature, 442, 1046-1049.

Monneveux, P., Ramírez, D. A., \& Pino, M. T. (2013). Drought tolerance in potato (S. tuberosum L.) Can we learn from drought tolerance research in cereals? Plant Science, 205-206 , 76-86.

Münzbergová, Z., Latzel, V., Šurinová, M., \& Hadincová, V. (2019). DNA methylation as a possible mechanism affecting ability of natural populations to adapt to changing climate. Oikos , 128 : 124-134.

Nakamura, M., \& Hennig, L. (2017). Inheritance of vernalization memory at FLOWERING LOCUS C during plant regeneration. Journal of Experimental Botany, 68, 2813-2819.

Pascual, J., Cañal, M. J., Correia, B., Escandon, M., Hasbún, R., Meijón, M., Pinto, G., \& Valledor, L. (2014). Can epigenetics help forest plants to adapt to climate change? // Alvarez-Venegas R, De la Peña C, CasasMollano J Armando eds. Epigenetics in Plants of Agronomic Importance: Fundamentals and Applications. Cham, Springer International Publishing, 125-146.

Paszkowski, J., \& Grossniklaus, U. (2011). Selected aspects of transgenerational epigenetic inheritance and resetting in plants. Current Opinion Plant Biology , 14 (2), 195-203.

Puy, J., Dvořáková, H., Carmona, C. P., de Bello, F., Hiiesalu, I., \& Latzel, V. (2018). Improved demethylation in ecological epigenetic experiments, Testing a simple and harmless foliar demethylation application. Methods in Ecology and Evolution, 9 , 744-753.

Racette, K., Rowland, D., Tillman, B., Erickson, J., Munoz, P., \& Vermerris, W. (2019). Transgenerational stress memory in seed and seedling vigor of peanut (Arachis hypogaea L.) varies by genotype. Environmental and Experimental Botany , 162, 541-549

Rahavi, S. M., \& Kovalchuk, I. (2013a). Changes in homologous recombination frequency in Arabidopsis thaliana plants exposed to stress depend on time of exposure during development and on duration of stress exposure. Physiology and Molecular Biology of Plants ,19 , 479-88.

Rahavi, S. M., \& Kovalchuk, I. (2013b). Transgenerational changes inArabidopsis thaliana in response to UV-C, heat and cold.Biocatalysis Agricutural Biotechnology, 2, 226-233.

Ramírez, D. A., Rolando, J. L., Yactayo, W., Monneveux, P., Mares, V., \& Quiroz, R. (2015), Improving potato drought tolerance through the induction of long-term water stress memory. Plant Science, 238 , 26-32.

Richards, C. L., Alonso, C., Becker, C., Bossdorf, O., Bucher, E., Colomé-Tatché, M., Durka, W., Engelhardt, J., Gaspar, B., Gogol-Döring, A., Grosse, I., van Gurp, T. P., Heer, K., Kronholm, I., Lampei, C., Latzel, V., Mirouze, M., Opgenoorth, L., Paun, O., Prohaska, S. J., Rensing, S. A., Stadler, P. F., Trucchi, E., Ullrich, K., \& Verhoeven, K. J. F. (2017). Ecological plant epigenetics: evidence from model and non-model species, and the way forward.Ecology Letters , 20, 1576-1590.

Sherwood, S., \& Fu, Q. (2014). Climate change. A drier future? Science ,343 , 737-739.

Shi, W., Chen, X., Gao, L., Xu, C. Y., Ou, X., Bossdorf, O., Yang, J. \& Geng, Y. (2019). Transient stability of epigenetic population differentiation in a clonal invader.Frontiers in Plant Science, , 9 ,1851.

Shock, C. C., Feibert, E. B. G., \& Saunders, L. D. (1998). Potato yield and quality response to deficit irrigation.HortScience ,33 (4), 655-659.

Tombesi, S., Frioni, T., Poni, S., \& Palliotti, A. (2018). Effect of water stress "memory" on plant behavior during subsequent drought stress.Environmental and Experimental Botany , 150 , 106-114.

Trewavas, A. (2014) Plant Behaviour and Intelligence. Oxford: Oxford University Press.

Verhoeven, K. J. F, van Dijk, P. J., \& Biere, A. (2010). Stress-induced DNA methylation changes and their heritability in asexual dandelions . New Phytologist , 185, 1108-1118. 
Verhoeven, K. J. F., \& van Gurp, T.P. (2012). Transgenerational effects of stress exposure on offspring phenotypes in apomictic dandelion. PLoS ONE , 6 , e38605.

Verhoeven, K. J. F., \& Preite, V. (2014). Epigenetic variation in asexually reproducing organisms.Evolution ,68 (3), 644-655.

Virlouvet, L., Avenson, T. J., Du, Q., Zhang, C., Liu, N., Fromm, M., Avramova, Z, \& Russo, S. E. (2018). Dehydration stress memory: gene networks linked to physiological responses during repeated stresses of Zea mays . Frontiers in Plant Science, $\mathbf{9}$.

Whittle, C. A., Otto, S. P., Johnston, M. O., \& Krochko, J. E. (2009). Adaptive epigenetic memory of ancestral temperature regime in Arabidopsis thaliana. Botany , 87, 650-657.

Xu, J. H, Tanino, K. K., \& Robinson, S. J. (2016). Stable epigenetic variants selected from an induced hypomethylatedFragaria vesca population. Frontiers in Plant science , 7 .

Table 1. Effects of genotype, time since last drought $(2 \mathrm{~W}, 4 \mathrm{~W}, 6 \mathrm{~W}, 8 \mathrm{~W}$ and Control) and 5-azaC treatment (control versus5-azaC) on parental biomass, size and growth rate of offspring. Values for $\mathrm{P}<0.05$ are in bold. Marginally significant $(\mathrm{P}<0.1)$ in italics .

\begin{tabular}{lllllll}
\hline & & Parental biomass & Parental biomass & Offspring biomass & Offspring biomass & Stolon \\
\hline & Df & $\mathrm{F}$ & $\mathrm{P}$ & $\mathrm{F}$ & $\mathrm{P}$ & $\mathrm{F}$ \\
Genotype & 2 & $\mathbf{3 2 . 6 5}$ & $<\mathbf{0 . 0 0 1}$ & 1.70 & 0.189 & $\mathbf{1 1 . 3}$ \\
Time since last drought $(\mathrm{T})$ & 4 & $\mathbf{1 6 6 . 3 2}$ & $<\mathbf{0 . 0 0 1}$ & $\mathbf{5 . 5 6}$ & $<\mathbf{0 . 0 0 1}$ & $\mathbf{9 . 8 8}$ \\
5 -azaC & 1 & 2.44 & 0.12 & 0.01 & 0.993 & 0.28 \\
Genotype $\times \mathrm{T}$ & 8 & 0.88 & 0.54 & 1.37 & 0.223 & 1.83 \\
Genotype $\times 5$-azaC & 2 & 1.01 & 0.37 & 0.58 & 0.563 & 0.08 \\
$\mathrm{~T} \times 5$-azaC & 4 & $\mathbf{2 . 6 2}$ & $\mathbf{0 . 0 4}$ & 2.35 & 0.060 & $\mathbf{3 . 6 7}$ \\
Genotype $\times \mathrm{T} \times 5$-azaC & 8 & 1.45 & 0.19 & 1.15 & 0.341 & $\mathbf{2 . 4}$ \\
\hline
\end{tabular}

Table 2. Effects of time since last drought ( $2 \mathrm{~W}, 4 \mathrm{~W}, 6 \mathrm{~W}, 8 \mathrm{~W}$ versus Control) on parental biomass, offspring biomass, node number of main stolon, number of side branches, growth rate of node number and side branches number of Trifolium repens across all three genotypes. Shown are means and SE.

Columns sharing the same letter are not significantly different from each other at $\mathrm{p}<0.05$.

\begin{tabular}{lllllll}
\hline & $2 \mathrm{~W}$ & $4 \mathrm{~W}$ & $6 \mathrm{~W}$ & $8 \mathrm{~W}$ & Control & \\
\hline Parental biomass /g & $12.36 \pm 3.21 \mathrm{c}$ & $14.50 \pm 4.13 \mathrm{c}$ & $14.52 \pm 3.07 \mathrm{c}$ & $18.60 \pm 6.09 \mathrm{~b}$ & $50.97 \pm 11.57 \mathrm{a}$ & $50.97 \pm 11.5$ \\
Offspring biomass / g & $1.97 \pm 0.15 \mathrm{ab}$ & $2.29 \pm 0.16 \mathrm{a}$ & $1.79 \pm 0.15 \mathrm{~b}$ & $1.61 \pm 0.15 \mathrm{bc}$ & $1.30 \pm 0.15 \mathrm{c}$ & $1.30 \pm 0.15 \mathrm{c}$ \\
Node number of main stolon & $12.42 \pm 0.54 \mathrm{~b}$ & $14.83 \pm 0.49 \mathrm{a}$ & $13.71 \pm 0.49 \mathrm{a}$ & $11.96 \pm 0.42 \mathrm{~b}$ & $11.88 \pm 1.13 \mathrm{~b}$ & $11.88 \pm 1.13 \mathrm{~b}$ \\
Number of side branches & $9.38 \pm 0.78 \mathrm{~b}$ & $11.83 \pm 0.71 \mathrm{a}$ & $9.79 \pm 0.71 \mathrm{~b}$ & $9.08 \pm 0.61 \mathrm{~b}$ & $6.88 \pm 1.63 \mathrm{~b}$ & $6.88 \pm 1.63 \mathrm{~b}$ \\
Growth rate - node no. & $1.02 \pm 0.00 \mathrm{~b}$ & $1.023 \pm 0.00 \mathrm{a}$ & $1.02 \pm 0.00 \mathrm{a}$ & $1.02 \pm 0.01 \mathrm{~b}$ & $1.02 \pm 0.01 \mathrm{a}$ & $1.02 \pm 0.01 \mathrm{a}$ \\
Growth rate - side branch no. & $1.04 \pm 0.02 \mathrm{~b}$ & $1.045 \pm 0.01 \mathrm{ab}$ & $1.05 \pm 0.01 \mathrm{ab}$ & $1.04 \pm 0.01 \mathrm{~b}$ & $1.05 \pm 0.02 \mathrm{a}$ & $1.05 \pm 0.02 \mathrm{a}$ \\
\hline
\end{tabular}

Figure legends:

Figure. 1 (a) Time schedule of the experiment. (b) Idealized scheme of T. repens plant developed after transplantation of parental cutting to a control environment in Offspring generation. Label: marked position of apical end of transplanted parental ramet. This enabled determination of parental ramet that developed prior transplantation and the offspring generation that developed after transplantation to control environment. 
Figure 2. Effects of time since last drought $(2 \mathrm{~W}, 4 \mathrm{~W}, 6 \mathrm{~W}, 8 \mathrm{~W}$ versus Control) and 5 -azaC treatment (control versus 5-azaC) on (a) node number of main stolon and (b) number of side branches of Trifolium repens. Shown are means and SE of all genotypes (main effect). Columns sharing the same letter are not significantly different from each other at $\mathrm{p}<0.05$.

Figure 3. Effects of time since last drought $(2 \mathrm{~W}, 4 \mathrm{~W}, 6 \mathrm{~W}, 8 \mathrm{~W}$ versus Control) on the offspring biomass of Trifolium repens. The graph shows mean $\pm \mathrm{SE}$ of all genotypes. Columns sharing the same letter are not significantly different from each other at $\mathrm{p}<0.05$.

Figure 4. Effects of time since last drought $(2 \mathrm{~W}, 4 \mathrm{~W}, 6 \mathrm{~W}, 8 \mathrm{~W}$ versus Control) and 5 -azaC agent treatment (control versus 5 -azaC) on growth rate of node number of main stolon of Trifolium repens. The graph shows mean $\pm \mathrm{SE}$ of all genotypes. Columns sharing the same letter are not significantly different from each other at $\mathrm{p}<0.05$. 
(a)

Time schedule of treatment applicantion

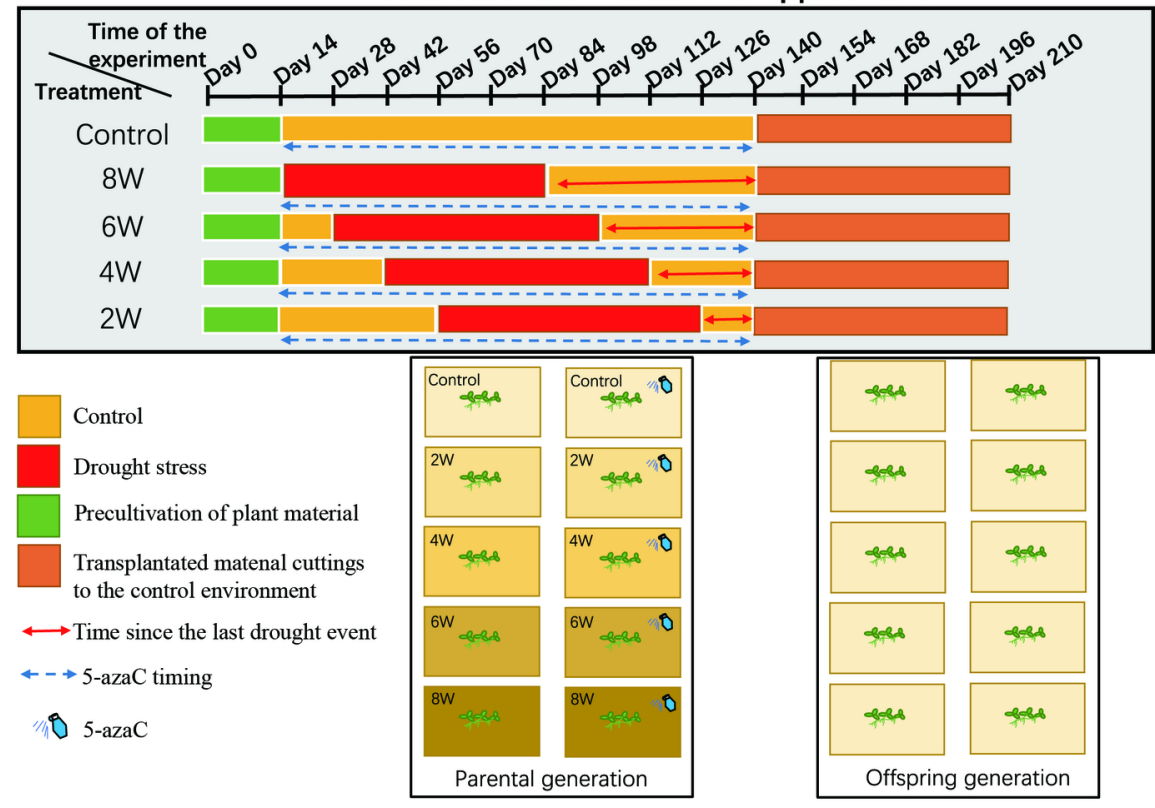

(b)

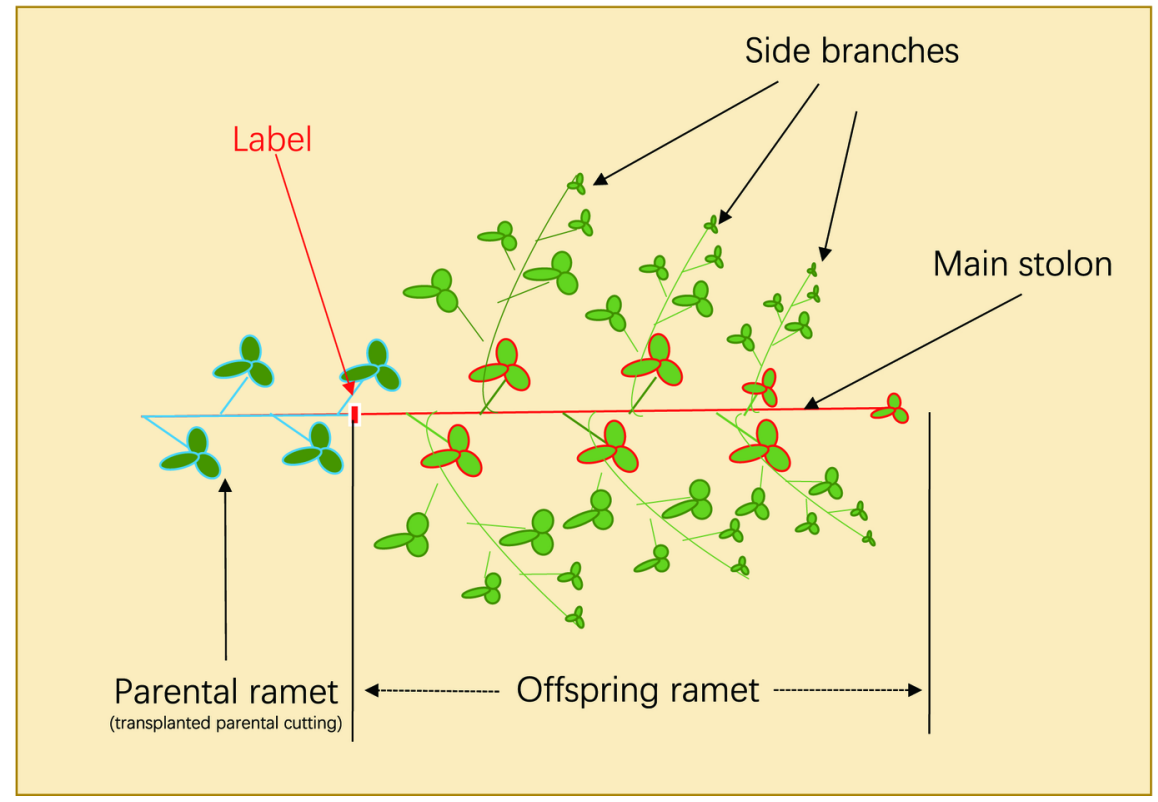



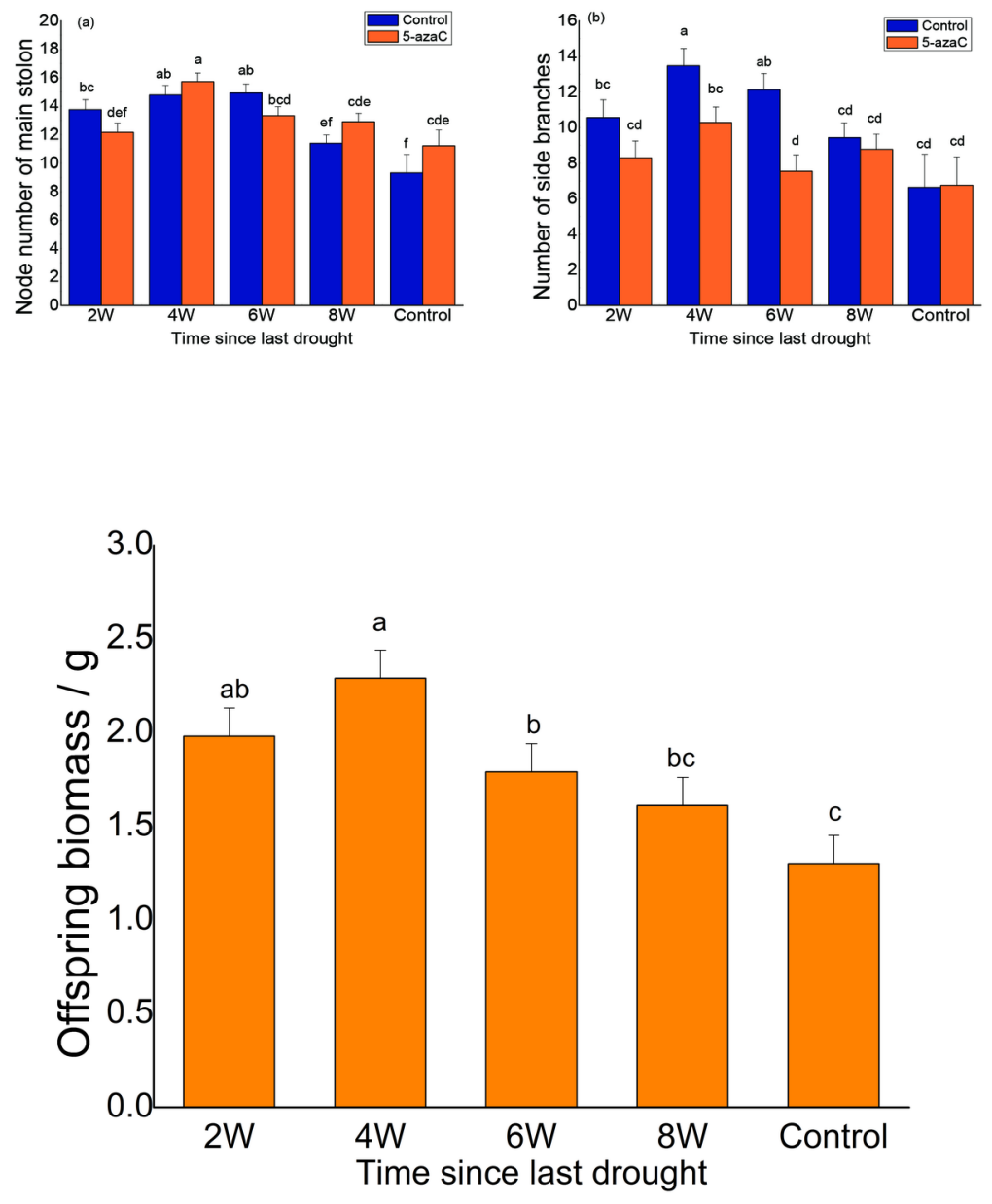


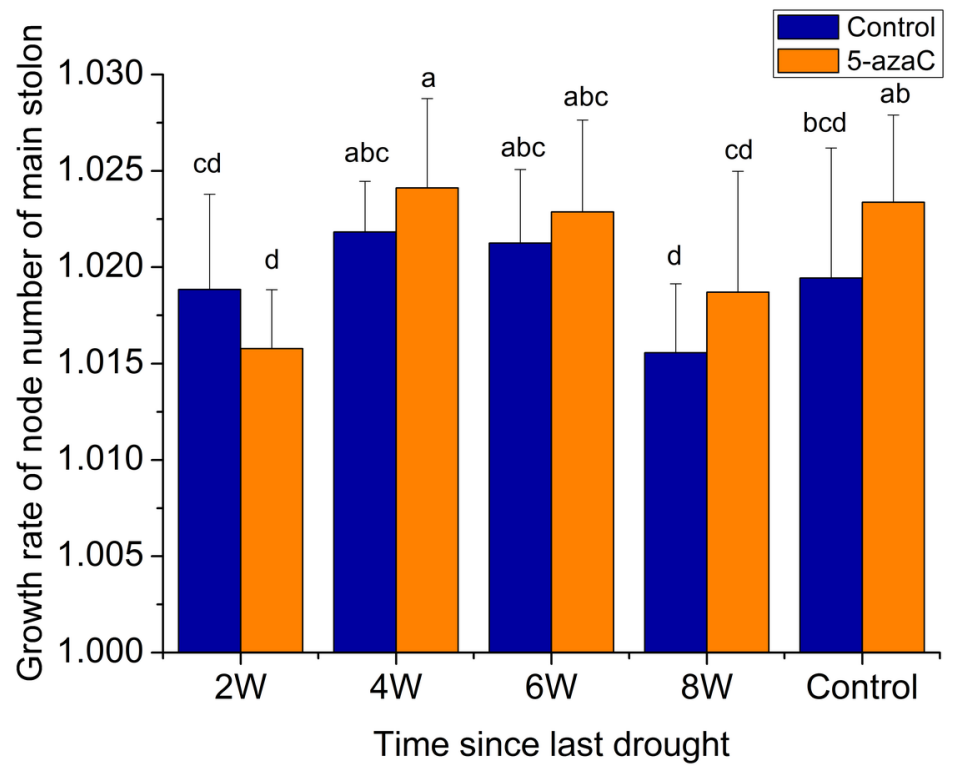

